J. Math. Soc. Japan

Vol. 49, No. 2, 1997

\title{
A diffusion process in a Brownian environment with drift*
}

\author{
By Kiyoshi KAWAZU and Hiroshi TANAKA
}

(Received Feb. 8, 1995)

\section{Introduction.}

Let $\boldsymbol{W}$ be the space of continuous functions $W$ defined in $\boldsymbol{R}$ and vanishing at the origin. Let $P$ be the Wiener measure on $\boldsymbol{W}$, namely, the probability measure on $\boldsymbol{W}$ such that $\{W(t), t \geqq 0, P\}$ and $\{W(-t), t \geqq 0, P\}$ are independent one-dimensional Brownian motions. Let $\Omega=C[0, \infty)$ and denote by $\omega(t)$ the value of a function $\boldsymbol{\omega}(\in \Omega)$ at time $t$. Given a sample function $W(\in \boldsymbol{W})$ and a nonnegative constant $\kappa$ we consider a probability measure $P_{W}^{x}$ on $\Omega$ such that $\left\{\boldsymbol{\omega}(t), t \geqq 0, P_{W}^{x}\right\}$ is a diffusion process with generator

$$
\mathcal{L}_{W}=\frac{1}{2} e^{W_{\kappa}(x)} \frac{d}{d x}\left(e^{-W_{\kappa}(x)} \frac{d}{d x}\right)=\frac{d x}{m_{W}(d x)} \cdot \frac{d x}{d S_{W}(x)}
$$

starting from $x$, where

$$
\begin{aligned}
& W_{\kappa}(x)=W(x)-\frac{1}{2} \kappa x, \\
& S_{W}(x)=\int_{0}^{x} e^{W_{\kappa}(y)} d y, \quad m_{W}(d x)=2 e^{-W_{\kappa}(x)} d x .
\end{aligned}
$$

It is well-known that a version of $\left\{\omega(t), t \geqq 0, P_{W}^{x}\right\}$ can be constructed from a Brownian motion by a scale-change and a time-change. When $W$ is considered random, $\{\omega(t), t \geqq 0\}$ is regarded as a process defined on the probability space $\left(\boldsymbol{W} \times \Omega, \mathscr{Q}^{x}\right)$ where $\mathscr{P}^{x}(d W d \boldsymbol{\omega})=P(d W) P_{W}^{x}(d \boldsymbol{\omega})$. We thus have a process $\boldsymbol{X}^{x}=$ $\left\{\boldsymbol{\omega}(t), t \geqq 0, \mathscr{Q}^{x}\right\}$ which, in this paper, is called a diffusion process in a Brownian environment with drift. The following intuitive description may suggest the name. The process $\boldsymbol{X}^{x}$ is obtained as a formal solution of the symbolic equation

$$
d X(t)=d B(t)-\frac{1}{2} W_{\kappa}^{\prime}(X(t)) d t
$$

* This work was partially supported by Grant-in Aid for Science (No. 07454034), Ministry of Education of Japan. 
where $B(t)$ is a Brownian motion independent of $W(\cdot)$ (however, note that (4) has no rigorous meaning).

When $\kappa>0 \boldsymbol{X}^{0}$ may be regarded as a diffusion model of a random walk in a random environment discussed by Kozlov [14], Solomon [18] and KestenKozlov-Spitzer [10]. When $\kappa=0 \quad \boldsymbol{X}^{0}$ is a diffusion model of Sinai's random walk in a random environment $\left([\mathbf{1 7 ]})\right.$. The asymptotic behavior of $\left\{\omega(t), \mathscr{P}^{0}\right\}$ as $t \rightarrow \infty$ when $\kappa=0$ was discussed by Schumacher [16] and Brox [1]. They showed that $\left\{\omega(t), \mathscr{Q}^{0}\right\}$ exhibits the same asymptotic behavior as Sinai's random walk, namely, that the limiting distribution of $(\log t)^{-2} \omega(t)$ as $t \rightarrow \infty$ exists (see also [2], [3], [8], [11], [19] for related works). When $\kappa>0$ (in particular when $0<\kappa<1$ ) it was an open problem to obtain results for $\boldsymbol{X}^{0}$ which are (or at least expected to be) similar to those of Kesten-Kozlov-Spitzer [10]. The purpose of the present paper is to give some answer to this problem.

Let $T_{x}=\inf \{t>0: \omega(t)=x\}, \bar{\omega}(t)=\max \{\omega(s): 0 \leqq s \leqq t\}$ and $\underline{\omega}(t)=\inf \{\omega(s): s \geqq t\}$. Then our result in the case $\kappa>0$ is the following.

THEOREM 1. (i) If $0<\kappa<1$, then

$$
\begin{aligned}
& \lim _{x \rightarrow \infty} \mathscr{Q}^{0}\left\{x^{-1 / \kappa} T_{x} \leqq t\right\}=F_{\kappa}(t), \quad t>0, \\
& \lim _{t \rightarrow \infty} \mathcal{P}^{0}\left\{t^{-\kappa} \bar{\omega}(t) \leqq x\right\}=\lim _{t \rightarrow \infty} \mathcal{P}^{0}\left\{t^{-\kappa} \omega(t) \leqq x\right\} \\
= & \lim _{t \rightarrow \infty} \mathcal{P}^{0}\left\{t^{-\kappa} \underline{\omega}(t) \leqq x\right\}=1-F_{\kappa}\left(x^{-1 / \kappa}\right), \quad x>0,
\end{aligned}
$$

where $F_{k}$ is the distribution function of a one-sided stable distribution with Laplace transform $\exp \left(-c \lambda^{\alpha}\right)$; the constant $c$ is given by

$$
c=\left\{2^{1-\kappa} \Gamma(\kappa) \int_{0}^{\infty} \frac{d x}{u(x)^{2}}\right\}^{-1}
$$

where $u(x)$ is the solution of

$$
\frac{d}{d M(x)} \frac{d}{d x} u=2 u, \quad u(0)=1, \quad u^{\prime}(0)=0,
$$

the function $M(x)$ being given in Lemma 1.

(ii) If $\kappa=1$, then

(7) $(x \log x)^{-1} T_{x}$ converges to 4 in probability with respect to $\mathscr{Q}^{0}$ as $x \rightarrow \infty$;

(8) each of $t^{-1}(\log t) \overline{\boldsymbol{\omega}}(t), t^{-1}(\log t) \omega(t)$ and $t^{-1}(\log t) \underline{\omega}(t)$ converges to $1 / 4$ in probability with respect to $\mathscr{Q}^{0}$ as $t \rightarrow \infty$.

(iii) If $\kappa>1$, then

$$
\lim _{x \rightarrow \infty} T_{x} / x=\frac{4}{\kappa-1}, \quad \mathscr{P}^{0} \text {-a.s. }
$$




$$
\lim _{t \rightarrow \infty} \omega(t) / t=\frac{\kappa-1}{4}, \quad \mathscr{Q}^{0}-a . s .
$$

The assertion (i) can be slightly strengthened as follows.

THEOREM 2. Let $0<\kappa<1$.

(i) The process $\left\{\lambda^{-1 / x} T_{\lambda x}, x \geqq 0, \mathscr{Q}^{0}\right\}$ converges to $\{L(x), x \geqq 0\}$ as $\lambda \rightarrow \infty$ in the sense of convergence of finite dimensional distributions, where $\{L(x), x \geqq 0\}$ is an increasing stable process with Laplace transform

$$
E\{\exp (-\xi L(1))\}=\exp \left(-c \xi^{\kappa}\right), \quad \xi \geqq 0 .
$$

(ii) The process $\left\{\lambda^{-\kappa} \omega(\lambda t), t \geqq 0, \mathscr{Q}^{0}\right\}$ converges to $\left\{L^{-1}(t), t \geqq 0\right\}$ as $\lambda \rightarrow \infty$ in the sense of convergence of finite dimensional distributions, where

$$
L^{-1}(t)=\inf \{x>0: L(x)>t\} \text {. }
$$

In the case of random walks, results similar to (5) and (6) were obtained by Kesten-Kozlov-Spitzer [10] and results similar to (7), (8), (9) and (10) by Solomon [18]. Our method of proving (9) and (10) is similar to that of [18] but as for (5), (6), (7) and (8) our method is different from either of [10] and [18]; it is based on Kotani's formula (see $\S 1$ ) which reduces our problem to the study of limiting behavior of another diffusion process described by a certain stochastic differential equation with non-random coefficients. In proving (5) and (6) we must also use Kasahara's continuity theorem ([7]) concerning Krein's correspondence $([6])$ between the $m$-measure and the spectral measure (or more precisely the $h$-function) of a one-dimensional diffusion operator.

AcKNowledgment. The authors wish to thank S. Kotani for valuable discussions on the subject and for permitting us to contain his formula (1.1) here.

\section{§1. Kotani's formula.}

The following formula was obtained by S. Kotani in 1988 in his study of the limiting distribution of $(\log t)^{-2} \bar{\omega}(t)$ in the case $\kappa=0$ (unpublished).

KOTANI's FoRmula. Let $\lambda>0$. Then for $t \geqq 0$

$$
E_{W}^{0}\left\{e^{-\lambda T_{t}}\right\}=\exp \left\{-\int_{0}^{t} U_{\lambda}(s) d s\right\}, \quad P \text {-a.s., }
$$

where $U_{\lambda}(t)$ is the unique stationary positive solution of

$$
d U_{\lambda}(t)=U_{\lambda}(t) d W(t)+\left\{2 \lambda+\frac{1-\kappa}{2} U_{\lambda}(t)-U_{\lambda}(t)^{2}\right\} d t .
$$


Proof. Taking an arbitrary but fixed $a>0$ we put for $0<t<a$

Then

$$
u(t)=1 / E_{W}^{0}\left\{e^{-\lambda T} t\right\}, \quad v(t)=E_{W}^{t}\left\{e^{-\lambda T_{a}}\right\} .
$$

$$
\begin{aligned}
E_{W}^{0}\left\{e^{-\lambda T_{a}}\right\} & =E_{W}^{0}\left\{e^{-\lambda T_{t}}\right\} E_{W}^{t}\left\{e^{-\lambda T_{a}}\right\} \\
& =v(t) / u(t), \quad P \text {-a.s.. }
\end{aligned}
$$

Since $\mathcal{L}_{W} v(t)=\lambda v(t), t<a, u(t)$ also satisfies $\mathcal{L}_{W} u(t)=\lambda u(t), t>0$, or equivalently

$$
d\left\{e^{-W_{\kappa}(t)} u^{\prime}(t)\right\}=2 \lambda e^{-W_{\boldsymbol{k}}(t)} u(t) d t, \quad t>0 .
$$

If we put $U_{\lambda}(t)=\{\log u(t)\}^{\prime}=u^{\prime}(t) / u(t)$, then $U_{\lambda}(t)>0, P$-a.s. Since $u(t)$ and $U_{\lambda}(t)$ are adapted to the filtration generated by $\{W(t)\}$, we can apply Itô's formula to compute the stochastic differential $d U_{\lambda}(t)$. Using [1.3) we have

$$
\begin{aligned}
d U_{\lambda}(t)= & d\left(u^{\prime}(t) u(t)^{-1}\right) \\
= & d\left(e^{-W_{\kappa}(t)} u^{\prime}(t) e^{W_{\kappa}(t)} u(t)^{-1}\right) \\
= & e^{W_{\kappa}(t)} u(t)^{-1} d\left(e^{-W_{\kappa}(t)} u^{\prime}(t)\right) \\
& +e^{-W_{\kappa}(t)} u^{\prime}(t) u(t)^{-1} d e^{W_{\kappa}(t)}-e^{-W_{\kappa}(t)} u^{\prime}(t) e^{W_{\kappa}(t)} u(t)^{-2} d u(t) \\
= & 2 \lambda d t+u^{\prime}(t) u(t)^{-1} d W_{\kappa}(t)+2^{-1} u^{\prime}(t) u(t)^{-1} d t-\left\{u^{\prime}(t) u(t)^{-1}\right\}^{2} d t \\
= & U_{\lambda}(t) d W(t)+\left\{2 \lambda+\frac{1-\kappa}{2} U_{\lambda}(t)-U_{\lambda}(t)^{2}\right\} d t .
\end{aligned}
$$

For $h>0$ we can write $u(t+h)=u(t) \tilde{u}(h)$ where

$$
\tilde{u}(h)=1 / E_{W}^{t}\left\{e^{-\lambda T_{t+h}}\right\} \stackrel{d}{=} u(h) ;
$$

in the above " $\stackrel{d}{=}$ " means the equality in distribution. Therefore

$$
\begin{aligned}
U_{\lambda}(t) & =u^{\prime}(t) u(t)^{-1}=\lim _{h \downarrow 0} \frac{\tilde{u}(h)-1}{h} \\
& \stackrel{d}{=} \lim _{h \downarrow 0} \frac{u(h)-1}{h}=u^{\prime}(0) \\
& =u^{\prime}(0) u(0)^{-1}=U_{\lambda}(0) .
\end{aligned}
$$

This implies that $U_{\lambda}(t)$ is a stationary solution of (1.2). The uniqueness of such a solution follows from Theorem 18 of Itô-Nisio [5].

\section{§ 2. Kasahara's continuity theorem for Krein's correspondence.}

Krein's theory of strings $[$ [6] has many applications to diffusion processes (e.g., see [12], [13], [20]). In this section we do not give the general theory but list some of the results of Kasahara [7] on Krein's correspondence that will be useful for our later discussions. For a general statement of Krein's 
correspondence theory it is convenient to consider inextensible measures (e.g., see [20]). From the view point of its application to the present paper, however, it is enough to consider simply Radon measures in $[0, \infty)$. Thus suppose we are given a Radon measure $m(d x)$ in $[0, \infty)$. We exclude the trivial case where $m(d x)=0$. The associated function $M(x)$ is defined by $M(x)=m([0, x))$ for $x>0$ and $M(0)=0$. Consider the generalized differential operator $\mathcal{L}=d / m(d x) \cdot d / d x$ and let $\varphi(x, \alpha)$ and $\phi(x, \alpha)$ be the solutions of $\mathcal{L} u=\alpha u$ with the initial conditions

$$
\begin{array}{ll}
u(0)=1, & u^{\prime}(0)=0, \\
u(0)=0, & u^{\prime}(0)=1,
\end{array}
$$

respectively. For $x>0 \varphi(x, \alpha)$ and $\phi(x, \alpha)$ satisfy

$$
\begin{aligned}
& \varphi(x, \alpha)=1+\alpha \iint_{0 \leqq z<y<x} \varphi(z, \alpha) m(d z) d y, \\
& \psi(x, \alpha)=x+\alpha \iint_{0 \leqq z<y<x} \psi(z, \alpha) m(d z) d y .
\end{aligned}
$$

The pair $\{\varphi(x, \alpha), \psi(x, \alpha)\}$ is called the system of fundamental solutions associated with $m(d x)$. It is known that for $\alpha>0$

$$
h(\alpha)=\lim _{x \rightarrow \infty} \psi(x, \alpha) / \varphi(x, \alpha)=\int_{0}^{\infty} \frac{d x}{\varphi(x, \alpha)^{2}}<\infty
$$

exists. The function $h(\alpha)$ is called the characteristic function of $m(d x)$ or of $M(x)$. The following (2.6), (2.7) and (2.8) are also known.

(2.6) The correspondence between $m(d x)$ and $h(\alpha)$ is one to one ([6], see also [13]).

(2.7) $a^{-1} h(c \alpha)$ is the characteristic function of $a c M(a x)$ for arbitrary positive constants $a$ and $c([7])$.

$$
\left\{\begin{array}{l}
\text { Assume that } \infty \text { is not regular for } \mathcal{L} \text {, namely, } \\
\text { that at least one of the integrals } \\
\iint_{0<y<x<\infty} m(d y) d x, \iint_{0<y<x<\infty} d y m(d x) \\
\text { diverges. Then for each } \alpha>0 \text { a positive decreasing solution } \\
u \text { of } \mathcal{L} u=\alpha u \text { with } u(0)=1 \text { is unique and expressed } \\
\text { as } u(x)=\varphi(x, \alpha)-\phi(x, \alpha) / h(\alpha)([4]) .
\end{array}\right.
$$

We now state 
Kasahara's Continuity Theorem $([7])$. Let $m_{n}(d x), n=0,1, \cdots$, be Radon measures in $[0, \infty)$ with associated functions $M_{n}(x)$, characteristic functions $h_{n}(\boldsymbol{\alpha})$ and systems of fundamental solutions $\left\{\varphi_{n}(x, \alpha), \psi_{n}(x, \alpha)\right\}$. Then the following statements are equivalent to each other.

(i) $M_{n}(x) \rightarrow M_{0}(x)$ as $n \rightarrow \infty$ at each continuity point $x$ of $M_{0}(\cdot)$.

(ii) For $x \geqq 0$ and $\alpha>0 \varphi_{n}(x, \alpha) \rightarrow \varphi_{0}(x, \alpha)$ as $n \rightarrow \infty$.

(iii) For $\alpha>0 h_{n}(\alpha) \rightarrow h(\alpha)$ as $n \rightarrow \infty$.

\section{$\S 3$. Proof of Theorem 1 in the case $0<\kappa<1$.}

Let $U_{\lambda}(t)$ be the diffusion process appearing in Kotani's formula and put

$$
V_{\lambda}(t)=\frac{1}{2 \lambda} U_{\lambda}(t) .
$$

Since $V_{\lambda}(t)$ satisfies the stochastic differential equation

$$
d V_{\lambda}(t)=V_{\lambda}(t) d W(t)+\left(1+\frac{1-\kappa}{2} V_{\lambda}(t)-2 \lambda V_{\lambda}(t)^{2}\right) d t
$$

the generator of the diffusion process $V_{\lambda}(t)$ is $\mathcal{L}_{\lambda}=d / m_{\lambda}(d x) \cdot d / d S_{\lambda}(x)$ where

$$
\begin{aligned}
& S_{\lambda}(x)=\int_{1}^{x} y^{x-1} \exp \left(\frac{2}{y}+4 \lambda y\right) d y, \\
& m_{\lambda}(d x)=2 x^{-\kappa-1} \exp \left(-\frac{2}{x}-4 \lambda x\right) d x .
\end{aligned}
$$

We also put

$$
\tilde{V}_{\lambda}(t)=V_{\lambda}\left(A_{\lambda}^{-1}(t)\right), \quad Y_{\lambda}(t)=S_{\lambda}\left(\tilde{V}_{\lambda}(t)\right),
$$

where $A_{\bar{\lambda}}^{-1}(t)$ is the inverse function of $A_{\lambda}(s)=\int_{0}^{s} V_{\lambda}(u) d u$. The generators of $\tilde{V}_{\lambda}(t)$ and $Y_{\lambda}(t)$ are denoted by $\widetilde{\mathcal{L}}_{\lambda}$ and $\mathcal{L}_{\lambda}^{0}$ respectively. $\mathcal{L}_{\lambda}^{0}$ is then given by $\mathcal{L}_{\lambda}^{0}=d / m_{\lambda}^{0}(d x) \cdot d / d x$ with

$$
m_{\lambda}^{0}(d x)=2 \theta_{\lambda}(x)^{-2 \kappa+1} \exp \left\{-\frac{4}{\theta_{\lambda}(x)}-8 \lambda \theta_{\lambda}(x)\right\} d x,
$$

where $\theta_{\lambda}(x)$ is the inverse function of $S_{\lambda}(\cdot)$. The path space representations of the diffusion processes with generators $\mathcal{L}_{\lambda}, \widetilde{\mathcal{L}}_{\lambda}$ and $\mathcal{L}_{\lambda}^{0}$ are denoted by $\left\{\omega(t), t \geqq 0, P_{\lambda}^{x}\right\},\left\{\omega(t), t \geqq 0, \tilde{P}_{\lambda}^{x}\right\}$ and $\left\{\omega(t), t \geqq 0, P_{\lambda}^{0, x}\right\}$, respectively. The expectations with respect to $P_{\lambda}^{x}, \widetilde{P}_{\lambda}^{x}$ and $P_{\lambda}^{0, x}$ will be denoted by $E_{\lambda}^{x}, \tilde{E}_{\lambda}^{x}$ and $E_{\lambda}^{0, x}$, respectively. We begin by proving the following lemma.

LEMMA 1. For any $x>0$ 


$$
\lim _{\lambda \downarrow 0} \lambda^{1-\kappa} M_{\lambda}\left(\lambda^{-\kappa} x\right)=M(x),
$$

where $M_{\lambda}(x)$ is the associated function of $m_{\lambda}^{0}(d x)$ and

$$
\begin{aligned}
& M(x)=2 \gamma\left(\rho^{-1}(x)\right), \\
& \gamma(x)=\int_{0}^{x} y^{-x} e^{-4 y} d y, \\
& \rho^{-1}(x) \text { is the inverse function of } \rho(x)=\int_{0}^{x} y^{\kappa-1} e^{4 y} d y .
\end{aligned}
$$

Proof. By an easy computation we have for $x>\lambda$

where

$$
S_{\lambda}\left(\lambda^{-1} x\right)=\lambda^{-\kappa} \rho_{\lambda}(x),
$$

$$
\rho_{\lambda}(x)=\int_{\lambda}^{x} y^{x^{-1}} \exp \left(\frac{2 \lambda}{y}+4 y\right) d y .
$$

It is also easy to see that $\rho_{\lambda}(x) \rightarrow \rho(x)$ as $\lambda \downarrow 0$ and hence $S_{\lambda}\left(\lambda^{-1} x\right) \sim \lambda^{-\kappa} \rho(x)$ as $\lambda \downarrow 0$. Therefore for any $\varepsilon \in(0, x) S_{\lambda}\left(\lambda^{-1}(x-\varepsilon)\right)<\lambda^{-\kappa} \rho(x)$ holds for all sufficiently small $\lambda>0$. In other words

$$
\lambda^{-1}(x-\varepsilon)<\theta_{\lambda}\left(\lambda^{-\kappa} \rho(x)\right) \text { for all sufficiently small } \lambda>0 \text {. }
$$

Similarly

$$
\lambda^{-1}(x+\varepsilon)>\theta_{\lambda}\left(\lambda^{-\kappa} \rho(x)\right) \text { for all sufficiently small } \lambda>0 \text {. }
$$

Next we note that

and hence

$$
M_{\lambda}(x)=2 \int_{1}^{\theta_{\lambda}(x)} z^{-2 \kappa+1} \exp \left(-\frac{4}{z}-8 \lambda z\right) S_{\lambda}^{\prime}(z) d z,
$$

$$
M_{\lambda}\left(\lambda^{-\kappa} \rho(x)\right)=2 \int_{1}^{\left.\theta \lambda^{(\lambda-\kappa} \rho(x)\right)} z^{-\kappa} \exp \left(-\frac{2}{z}-4 \lambda z\right) d z .
$$

This combined with (3.5) and (3.6) yields

$$
\begin{aligned}
2 \int_{1}^{\lambda-1(x-\varepsilon)} z^{-\kappa} \exp \left(-\frac{2}{z}-4 \lambda z\right) d z & <M_{\lambda}\left(\lambda^{-\kappa} \rho(x)\right) \\
& <2 \int_{1}^{\lambda^{-1}(x+\varepsilon)} z^{-\kappa} \exp \left(-\frac{2}{z}-4 \lambda z\right) d z
\end{aligned}
$$

for all sufficiently small $\lambda>0$. Since

$$
2 \int_{1}^{\lambda-1} z^{-\kappa} \exp \left(-\frac{2}{z}-4 \lambda z\right) d z=2 \lambda^{x-1} \gamma_{\lambda}(x)
$$

where

$$
\gamma_{\lambda}(x)=\int_{\lambda}^{x} y^{-\kappa} \exp \left(-\frac{2 \lambda}{y}-4 y\right) d y,
$$


(3.7) yields

$$
\gamma_{\lambda}(x-\varepsilon)<M_{\lambda}\left(\lambda^{-\kappa} \rho(x)\right) /\left(2 \lambda^{\kappa-1}\right)<\gamma_{\lambda}(x+\varepsilon),
$$

which again yields

$$
\lim _{\lambda \downarrow 0} M_{\lambda}\left(\lambda^{-\kappa} \rho(x)\right) /\left(2 \lambda^{\kappa-1}\right)=\gamma(x),
$$

because $\gamma_{\lambda}(x) \rightarrow \gamma(x)$ as $\lambda \downarrow 0$. Taking $\rho^{-1}(x)$ instead of $x$ we obtain

$$
\lim _{\lambda \downarrow 0} M_{\lambda}\left(\lambda^{-x} x\right) /\left(2 \lambda^{x-1}\right)=\gamma\left(\rho^{-1}(x)\right),
$$

which proves Lemma 1 .

By virtue of Kasahara's continuity theorem and (2.7) Lemma 1 yields the following lemma concerning the characteristic functions $h_{\lambda}(\alpha)$ and $h(\alpha)$ of $M_{\lambda}(x)$ and $M(x)$ respectively.

LEMMA 2. $\lim _{\lambda \downarrow 0} \lambda^{\kappa} h_{\lambda}(\lambda \alpha)=h(\alpha), \alpha>0$.

Let $\tau=\inf \{t>0: \omega(t)=1\}$. Then for $x>0$ and $\alpha \geqq 0$

$$
E_{\lambda}^{x}\left\{\exp \left(-\alpha \lambda \int_{0}^{\tau} \omega(s) d s\right)\right\}=\tilde{E}_{\lambda}^{x}\left\{e^{-\alpha \lambda \tau}\right\}=E_{\lambda}^{0, S_{\lambda}(x)}\left\{e^{-\alpha \lambda \sigma}\right\},
$$

where $\sigma=\inf \{t>0: \omega(t)=0\}$. For a given $a>0$ determine $a_{\lambda}(>1)$ by $S_{\lambda}\left(a_{\lambda}\right)=a$. Also let

$$
S(x)=\int_{1}^{x} y^{k-1} \exp \left(\frac{2}{y}\right) d y,
$$

and determine $a_{0}(>1)$ by $S\left(a_{0}\right)=a$. Then $a_{\lambda} \uparrow a_{0}$ as $\lambda \downarrow 0$.

LEMMA 3. If $0<\kappa<1$, then for $\alpha>0$

$$
1-E_{\lambda}^{a}\left\{\exp \left(-\alpha \lambda \int_{0}^{\tau} \omega(s) d s\right)\right\} \sim c(a, \alpha) \lambda^{\kappa} \text { as } \lambda \downarrow 0,
$$

where

$$
c(a, \alpha)=a / h(\alpha) .
$$

Proof. Since $\infty$ is not regular for $\mathcal{L}_{\lambda}^{0},(2.8)$ implies that for each $\alpha>0$ a positive decreasing solution $u_{\lambda}(\cdot, \alpha)$ of $\mathcal{L}_{\lambda}^{0} u=\alpha u$ with $u(0)=1$ is unique and expressed as

$$
u_{\lambda}(x, \alpha)=\varphi_{\lambda}(x, \alpha)-\phi_{\lambda}(x, \alpha) / h_{\lambda}(\alpha),
$$

where the pair $\left\{\varphi_{\lambda}(x, \alpha), \phi_{\lambda}(x, \alpha)\right\}$ is the system of fundamental solutions associated with $m_{\lambda}^{0}(d x)$. By (3.8) we have

$$
E_{\lambda}^{a \lambda}\left\{\exp \left(-\alpha \lambda \int_{0}^{\tau} \omega(s) d s\right)\right\}=u_{\lambda}(a, \alpha \lambda)
$$


By making use of (2.3) and (2.4) with $m(d z)$ replaced by $m_{\lambda}^{0}(d z)$ we can easily prove that

$$
\begin{gathered}
\varphi_{\lambda}(a, \alpha \lambda)-1=2 \alpha \lambda \int_{0}^{a} d x \int_{1}^{\theta_{\lambda}(x)} y^{-\kappa} \exp \left(-\frac{2}{y}-4 \lambda y\right) d y+o(\lambda), \\
\phi_{\lambda}(a, \alpha \lambda)=a+O(\lambda),
\end{gathered}
$$

as $\lambda \downarrow 0$. Denoting by $\theta(x)$ the inverse function of $S(\cdot)$, we have

$$
\lim _{\lambda \downarrow 0} \frac{\varphi_{\lambda}(a, \alpha \lambda)-1}{\alpha \lambda}=2 \int_{0}^{a} d x \int_{1}^{\theta(x)} y^{-\kappa} \exp \left(-\frac{2}{y}\right) d y=\text { const. . }
$$

From (3.12) and (3.11) we have

$$
1-E_{\lambda}^{a \lambda}\left\{\exp \left(-\alpha \lambda \int_{0}^{\tau} \omega(s) d s\right)\right\}=1-\varphi_{\lambda}(a, \alpha \lambda)+\phi_{\lambda}(a, \alpha \lambda) / h_{\lambda}(\alpha \lambda),
$$

which combined with (3.15), (3.14) and Lemma 2 finally implies (3.9).

The idea of the proof of (5) of Theorem 1 is as follows. We want to compute

$$
\lim _{\lambda \downarrow 0} \mathcal{E}^{0}\left\{\exp \left(-\lambda T_{\lambda-\kappa_{t}}\right)\right\}
$$

which, by virtue of Kotani's formula, equals

$$
\begin{aligned}
& \lim _{\lambda \downarrow 0} E\left\{\exp \left(-\int_{0}^{\lambda-\kappa_{t}} U_{\lambda}(s) d s\right)\right\}, \\
= & \lim _{\lambda \downarrow 0} E\left\{\exp \left(-2 \lambda \int_{0}^{\lambda-\kappa_{t}} V_{\lambda}(s) d s\right)\right\} \\
= & \lim _{\lambda \downarrow 0} E_{\lambda}^{\mu}\left\{\exp \left(-2 \lambda \int_{0}^{\lambda-\kappa_{t}} \omega(s) d s\right)\right\},
\end{aligned}
$$

where $E_{\lambda}^{\mu_{\lambda}}$ denotes the expectation with respect to $P_{\lambda}^{\mu_{\lambda}}=\int \mu_{\lambda}(d x) P_{\lambda}^{x}, \mu_{\lambda}$ being the invariant probability measure of the diffusion process with generator $\mathcal{L}_{\lambda}$. We first compute

$$
\lim _{\lambda \downarrow 0} E_{\lambda}^{a}\left\{\left\{\exp \left(-2 \lambda \int_{0}^{\lambda-\kappa_{t}} \omega(s) d s\right)\right\}\right.
$$

with the starting point $a_{\lambda}$ defined by $S_{\lambda}\left(a_{\lambda}\right)=a$, by showing that $\int_{0}^{\lambda-\kappa t} \omega(s) d s$ can be approximated by $\Sigma \int_{\sigma_{k-1}}^{\tau_{k}} \omega(s) d s$ where $\sigma_{k}$ and $\tau_{k}$ are defined as follows:

$$
\begin{array}{ll}
\sigma_{0}=0, \quad \tau_{k}=\inf \left\{t>\sigma_{k-1}: \omega(t)=1\right\}, & k \geqq 1, \\
\sigma_{k}=\inf \left\{t>\tau_{k}: \omega(t)=a_{\lambda}\right\}, & k \geqq 1 .
\end{array}
$$

Note that $\sigma_{k}-\sigma_{k-1}, k \geqq 1$, are i.i.d. random variables. 
LEMMA 4. (i) $m_{\lambda}=E_{\lambda}^{a} \lambda\left\{\sigma_{1}\right\}=a m_{\lambda}\left(\boldsymbol{R}^{+}\right)<\infty, \lambda \geqq 0$, where $\boldsymbol{R}^{+}=(0, \infty)$.

(ii) For any $\varepsilon>0$ and $\lambda \geqq 0$

$$
P_{\lambda}^{a \lambda}\left\{\left|\frac{\sigma_{n}}{n}-m_{\lambda}\right|>\varepsilon\right\} \rightarrow 0 \text { as } n \rightarrow \infty,
$$

and the convergence is uniform in $\lambda \in[0,1]$.

Proof. For $b>0$ and $x>0$ let $I=(x, b)$ and $J=(0, y)$ or $I=(b, x)$ and $J=$ $(y, \infty)$ according as $0<x<b$ or $0<b<x$. Let $\sigma=\inf \{t>0: \omega(t)=b\}$. Then it is well-known that

$$
E_{\lambda}^{x}\{\sigma\}=\int_{I} d S_{\lambda}(y) \int_{J} m_{\lambda}(d z)
$$

holds (note that $-S_{\lambda}(0)=S_{\lambda}(\infty)=\infty$ is also taken into account in deriving the above formula). By virtue of [(3.18) we can easily compute $m_{\lambda}=E_{\lambda}^{a}{ }^{\lambda}\left\{\tau_{1}\right\}+$ $E_{\lambda}^{a \lambda}\left\{\sigma_{1}-\tau_{1}\right\}$, obtaining (i). The assertion (3.17) is nothing but the law of large numbers for i.i.d. random variables. Only the uniform convergence needs proof for which it is enough to verify the uniform integrability of $\left\{\sigma_{1}, P_{\lambda}^{a}, 0 \leqq \lambda \leqq 1\right\}$, namely,

$$
\lim _{N \rightarrow \infty} \sup _{0 \leq \lambda \leq 1} \int_{\left(\sigma_{1}>N\right)} \sigma_{1} d P_{\lambda}^{a \lambda}=0 .
$$

We use the fact that the diffusion process $\left\{\omega(t), t \geqq 0, P_{\lambda}^{a}{ }_{\lambda}\right\}$ can also be realized as a solution of the stochastic differential equation

$$
d V(t)=V(t) d W(t)+\left\{1+\frac{1-\kappa}{2} V(t)-2 \lambda V(t)^{2}\right\} d t
$$

with $V(0)=a_{\lambda}$. Then a comparison theorem in stochastic differential equations implies that the solution of $(3.20)$ lies below the solution of $(3.20)$ with $\lambda=0$. From this observation we see that

$$
P_{\lambda}^{a \lambda}\left\{\tau_{1}>N\right\} \leqq P_{0}^{a_{0}}\left\{\tau_{1}>N\right\},
$$

and by a similar argument

$$
P_{\lambda}^{a_{\lambda} \lambda}\left\{\sigma_{1}-\tau_{1}>N\right\} \leqq P_{1}^{1}\{\sigma>N\},
$$

where $\sigma=\inf \left\{t>0: \omega(t)=a_{0}\right\}$. Thus (3.19) follows from (3.21) and (3.22). The proof of the lemma is finished.

We are now in the final stage of the proof of Theorem 1 in the case $0<\kappa<1$. For fixed $t>0$ and small $\varepsilon>0$ we put

$$
n_{1}(\lambda)=\left[\lambda^{-\kappa} t(1-\varepsilon) / m_{0}\right], \quad n_{2}(\lambda)=\left[\lambda^{-\kappa} t(1+\varepsilon) / m_{0}\right] .
$$

Then the uniform convergence of (3.17) implies 


$$
\lim _{\lambda \downarrow 0} P_{\lambda}^{a \lambda}\left\{\left|\frac{\sigma_{n_{i}(\lambda)}}{n_{i}(\lambda)}-m_{\lambda}\right|>m_{0} \varepsilon\right\}=0, \quad i=1,2 .
$$

Since $m_{\lambda} \rightarrow m_{0}$ as $\lambda \downarrow 0$ we have

$$
\lim _{\lambda \downarrow 0} P_{\lambda}^{a} \lambda\left\{\left|\frac{\sigma_{n_{i}(\lambda)}}{n_{i}(\lambda)}-m_{0}\right|>m_{0} \varepsilon\right\}=0, \quad i=1,2,
$$

and in particular

$$
\lim _{\lambda \downarrow 0} P_{\lambda}^{a}\left\{\sigma_{n_{1}(\lambda)}<n_{1}(\lambda) m_{0}(1+\varepsilon)\right\}=1 .
$$

But $n_{1}(\lambda) m_{0}(1+\varepsilon)<\lambda^{-\kappa} t$ for all sufficiently small $\lambda>0$ and hence

$$
\lim _{\lambda \downarrow 0} P_{\lambda}^{a \lambda}\left\{\sigma_{n_{1}(\lambda)}<\lambda^{-\kappa} t\right\}=1 \text {. }
$$

Similarly we have

$$
\lim _{\lambda \downarrow 0} P_{\lambda}^{a}\left\{\sigma_{n_{2}(\lambda)}>\lambda^{-\kappa} t\right\}=1
$$

Next we put

$$
Y_{k}=\int_{\sigma_{k-1}}^{\tau_{k}} \omega(s) d s, \quad Z_{k}=\int_{\tau_{k}}^{\sigma_{k}} \omega(s) d s, \quad k \geqq 1 .
$$

Then on the event $A_{\lambda}=\left\{\sigma_{n_{1}(\lambda)}<\lambda^{-\kappa} t<\sigma_{n_{2}(\lambda)}\right\}$

$$
\sum_{k=1}^{n_{1}(\lambda)}\left(Y_{k}+Z_{k}\right)<\int_{0}^{\lambda-k_{t}} \omega(s) d s<\sum_{k=1}^{n_{2(\lambda)}}\left(Y_{k}+Z_{k}\right)
$$

holds and hence

$$
\begin{aligned}
& E_{\lambda}^{a \lambda}\left\{\exp \left(-2 \lambda \sum_{k=1}^{n_{2}(\lambda)}\left(Y_{k}+Z_{k}\right)\right) ; A_{\lambda}\right\} \\
\leqq & E_{\lambda}^{a}\left\{\exp \left(-2 \lambda \int_{0}^{\lambda-\kappa_{t}} \omega(s) d s\right) ; A_{\lambda}\right\} \\
\leqq & E_{\lambda}^{a}\left\{\exp \left(-2 \lambda \sum_{k=1}^{n_{1}(\lambda)}\left(Y_{k}+Z_{k}\right)\right) ; A_{\lambda}\right\},
\end{aligned}
$$

where the notation $E_{2}^{a}\{X ; A\}$ stands for the integral of $X$ over $A$ with respect to $P_{\lambda}^{a \lambda}$. Since $P_{\lambda}^{a_{\lambda}}\left\{A_{\lambda}\right\} \rightarrow 1$ by (3.24) and (3.25),

$$
\begin{aligned}
& E_{\lambda}^{a}\left\{\exp \left(-2 \lambda \sum_{k=1}^{n_{2}(\lambda)}\left(Y_{k}+Z_{k}\right)\right)\right\} \\
\leqq & E_{\lambda}^{a}\left\{\exp \left(-2 \lambda \int_{0}^{\lambda-\kappa_{t}} \omega(s) d s\right)\right\}+o(1) \\
\leqq & E_{\lambda}^{a}\left\{\exp \left(-2 \lambda \sum_{k=1}^{n_{1}(\lambda)}\left(Y_{k}+Z_{k}\right)\right)\right\}+o(1),
\end{aligned}
$$

where $o(1)$ indicates a term which tends to 0 as $\lambda \downarrow 0$. We now compute 


$$
\begin{aligned}
& \lim _{\lambda \downarrow 0} E_{\lambda}^{a \lambda}\left\{\exp \left(-2 \lambda \sum_{k=1}^{n_{i}(\lambda)}\left(Y_{k}+Z_{k}\right)\right)\right\} \\
= & \lim _{\lambda \downarrow 0} E_{\lambda}^{a \lambda}\left\{\exp \left(-2 \lambda Y_{1}\right)\right\}^{n_{i}(\lambda)} \cdot E_{\lambda}^{a \lambda}\left\{\exp \left(-2 \lambda Z_{1}\right)\right\}^{{ }^{n} i(\lambda)} .
\end{aligned}
$$

By Lemma 3 we have

$$
\begin{aligned}
\lim _{\lambda \downarrow 0}\left[E_{\lambda}^{a} \lambda\left\{\exp \left(-2 \lambda Y_{1}\right)\right\}\right]^{n_{1}(\lambda)} & =\lim _{\lambda \downarrow 0}\left\{1-c(a, 2) \lambda^{\alpha}\right\}^{n_{1}(\lambda)} \\
& =\exp \left\{-c(a, 2) t(1-\varepsilon) / m_{0}\right\} .
\end{aligned}
$$

Similarly we have

$$
\lim _{\lambda \downarrow 0} E_{\lambda}^{a}\left\{\exp \left(-2 \lambda \sum_{k=1}^{n_{2}(\lambda)} Y_{k}\right)\right\}=\exp \left\{-c(a, 2) t(1+\varepsilon) / m_{0}\right\} .
$$

On the other hand, we have

$$
2 \lambda \sum_{k=1}^{n_{i}(\lambda)} Z_{k}<2 \lambda a_{\lambda} \sigma_{n_{i}(\lambda)}<2 a_{\lambda} \lambda^{1-\kappa} t(1+\varepsilon) m_{0}^{-1} \sigma_{n_{i}(\lambda)} / n_{i}(\lambda)
$$

which tends to 0 in probability as $\lambda \downarrow 0$ by virtue of $0<\kappa<1$ and (3.23). Therefore

$$
\lim _{\lambda \downarrow 0} E_{\lambda}^{a} \lambda\left\{\exp \left(-2 \lambda \sum_{k=1}^{n_{i}(\lambda)} Z_{k}\right)\right\}=1 .
$$

From (3.26), (3.27), (3.28) and (3.29) we have for any $\varepsilon>0$

which implies

$$
\begin{aligned}
\exp \left\{-c(a, 2) t(1+\varepsilon) / m_{0}\right\} & \leqq \liminf _{\lambda \downarrow 0} E_{\lambda}^{a}\left\{\exp \left(-2 \lambda \int_{0}^{\lambda-\kappa_{t}} \omega(s) d s\right)\right\} \\
& \leqq \limsup _{\lambda \downarrow 0} E_{\lambda}^{a \lambda}\left\{\exp \left(-2 \lambda \int_{0}^{\lambda-\kappa_{t}} \omega(s) d s\right)\right\} \\
& \leqq \exp \left\{-c(a, 2) t(1-\varepsilon) / m_{0}\right\}
\end{aligned}
$$

$$
\lim _{\lambda \downarrow 0} E_{\lambda}^{a_{\lambda}}\left\{\exp \left(-2 \lambda \int_{0}^{\lambda-\kappa_{t}} \omega(s) d s\right)\right\}=\exp \left\{-c(a, 2) t / m_{0}\right\} .
$$

LEMMA 5: For any $t>0$

$$
\lim _{\lambda \downarrow 0} E\left\{\exp \left(-\int_{0}^{\lambda-\kappa_{t}} U_{\lambda}(s) d s\right)\right\}=\exp \left\{-c(a, 2) t / m_{0}\right\} .
$$

Proof. Note that by (3.16) the left hand side of (3.31) equals

$$
\lim _{\lambda \downarrow 0} E_{\lambda}^{\mu} \lambda\left\{\exp \left(-2 \lambda \int_{0}^{\lambda-\kappa_{t}} \omega(s) d s\right)\right\}
$$

Let $\sigma_{0}=\inf \left\{t>0: \omega(t)=a_{\lambda}\right\}$ and put $\Gamma_{u}=\left\{\sigma_{0}<u\right\}$. Then using the strong Markov property we see that 


$$
E_{\lambda}^{\mu \lambda}\left\{\exp \left(-2 \lambda \int_{0}^{\lambda-\kappa_{t}} \omega(s) d s\right) ; \Gamma_{u}\right\}
$$

is bounded from below by

$$
E_{\lambda}^{\mu_{\lambda}}\left\{\exp \left(-2 \lambda \int_{0}^{\sigma_{0}} \omega(s) d s\right) ; \Gamma_{u}\right\} \cdot E_{\lambda}^{a \lambda}\left\{\exp \left(-2 \lambda \int_{0}^{\lambda-\kappa_{t}} \omega(s) d s\right)\right\}
$$

and is bounded from above by

$$
E_{\lambda}^{a} \lambda\left\{\exp \left(-2 \lambda \int_{0}^{\lambda-\kappa\left(t-\lambda^{\kappa} u\right)} \omega(s) d s\right)\right\}
$$

Taking into account of the fact that $P_{\lambda}^{\mu}\left\{\Gamma_{u}\right\} \rightarrow 1$ as $u \rightarrow \infty$ uniformly in $\lambda \in(0,1)$ and also of (3.30), we first left $\lambda \downarrow 0$ and then $u \uparrow \infty$. As a result we obtain (3.31).

The proof of (5) in Theorem 1 is now completed as follows. By Kotani's formula we have for $\xi>0$

$$
\begin{aligned}
\mathcal{E}^{0}\left\{\exp \left(-\xi T_{x} / x^{1 / \kappa}\right)\right\} & =E\left\{\exp \left(-\int_{0}^{x} U_{\xi x^{-1 / \kappa}}(s) d s\right)\right\} \\
& =E\left\{\exp \left(-\int_{0}^{\lambda-\kappa_{t}} U_{\lambda}(s) d s\right)\right\},
\end{aligned}
$$

where we put $t=\xi^{\kappa}$ and $\lambda=\xi x^{-1 / \kappa}$. Letting $x \rightarrow \infty$ (so $\lambda \downarrow 0$ ) we obtain

$$
\lim _{x \rightarrow \infty} \mathcal{E}^{0}\left\{\exp \left(-\xi T_{x} / x^{1 / \kappa}\right)\right\}=\exp \left\{-c(a, 2) t / m_{0}\right\}=e^{-c t}=e^{-c \xi \kappa},
$$

where $c=\left\{2^{1-\kappa} \Gamma(\kappa) h(2)\right\}^{-1}$, because $c(a, 2)=a / h(2)$ and

$$
m_{0}=a m_{0}\left(\boldsymbol{R}^{+}\right)=2 a \int_{0}^{\infty} x^{-\kappa-1} e^{-2 / x} d x=2^{1-\kappa} \Gamma(\kappa) a
$$

by (3.10) and (i) of Lemma 4 ,

Finally we prove (6). Clearly we see that for any $u>0, y>0$ and $t>0$

$$
\begin{aligned}
\left\{T_{u} \geqq t\right\} & \subset\{\bar{\omega}(t) \leqq u\} \subset\{\boldsymbol{\omega}(t) \leqq u\} \subset\{\underline{\omega}(t) \leqq u\} \\
& \subset\left\{T_{u+y} \geqq t\right\} \cup\left\{\inf _{s \geqq T_{u+y}} \omega(s)-(u+y) \leqq-y\right\} .
\end{aligned}
$$

We notice that

$$
\lim _{y \rightarrow \infty} \mathcal{Q}^{0}\left\{\inf _{s \geq T} \omega(s)-(u+y) \leqq-y\right\}=\lim _{y \rightarrow \infty} \mathscr{P}^{0}\left\{\inf _{s \geq 0} \omega(s) \leqq-y\right\}=0
$$

since $\omega(s) \rightarrow \infty$ as $s \uparrow \infty, \mathscr{Q}^{0}$-a.s.. Therefore we have for all $x>0$

$$
\begin{gathered}
\mathscr{P}^{0}\left\{T_{t^{\kappa} x} \geqq t\right\} \leqq \mathscr{P}^{0}\left\{\bar{\omega}(t) \leqq t^{\kappa} x\right\} \leqq \mathscr{P}^{0}\left\{\omega(t) \leqq t^{\kappa} x\right\} \\
\leqq \mathscr{P}^{0}\left\{\underline{\omega}(t) \leqq t^{\kappa} u\right\} \leqq \mathcal{P}^{0}\left\{T_{t^{\kappa} x+y} \geqq t\right\}+\mathscr{P}^{0}\left\{\inf _{s \geq 0} \omega(s) \leqq-y\right\} .
\end{gathered}
$$


For any sufficiently large fixed $y$, the result (5) ensures

$$
\begin{aligned}
\lim _{t \rightarrow \infty} \mathscr{P}^{0}\left\{T_{t^{\kappa} x} \geqq t\right\} & =\lim _{t \rightarrow \infty} \mathscr{P}^{0}\left\{T_{t^{\kappa} x+y} \geqq t\right\} \\
& =\lim _{v \rightarrow \infty} \mathscr{P}^{0}\left\{v^{-1 / \kappa} T_{v} \geqq x^{-1 / \kappa}\right\}=1-F_{\kappa}\left(x^{-1 / \kappa}\right),
\end{aligned}
$$

which combined with (3.32) and (3.33) proves (6).

\section{§4. Proof of Theorem 2.}

For the proof of (i) it is enough to show that

(4.1) $\lim _{\lambda \rightarrow \infty} \mathcal{Q}^{0}\left\{\exp \left(-\sum_{k=1}^{n} \xi_{k} \lambda^{-1 / \kappa}\left(T_{\lambda x_{k}}-T_{\lambda x_{k-1}}\right)\right)\right\}=\exp \left\{-c \sum_{k=1}^{n}\left(x_{k}-x_{k-1}\right) \xi_{k}^{k}\right\}$

for any $\xi_{1}, \xi_{2}, \cdots, \xi_{n} \geqq 0$ and $0=x_{0}<x_{1}<\cdots<x_{n}$. Take an $\varepsilon$ such that $0<\varepsilon<$ $\min \left\{x_{k}-x_{k-1}: 1 \leqq k \leqq n\right\}$ and let us prove first that

(4.2) $\lim _{\lambda \rightarrow \infty} \mathcal{E}^{0}\left\{\exp \left(-\sum_{k=1}^{n} \xi_{k} \lambda^{-1 / \kappa}\left(T_{\lambda\left(x_{k}-\varepsilon\right)}-T_{\lambda x_{k-1}}\right)\right)\right\}=\exp \left\{-c \sum_{k=1}^{n}\left(x_{k}-x_{k-1}-\varepsilon\right) \xi_{k}^{\kappa}\right\}$.

In what follows $T_{x}=T_{x}(\omega)$ denotes the first passage time inf $\{t>0: \omega(t)=x\}$ where $x$ may lie either to the right or to the left of $\omega(0)$. If we put

$$
\begin{aligned}
& F_{\lambda, k}=E_{W}^{\lambda x_{k-1}}\left\{\exp \left(-\xi_{k} \lambda^{-1 / \kappa} T_{\lambda\left(x_{k}-\varepsilon\right)}\right) ; T_{\lambda\left(x_{k}-\varepsilon\right)}<T_{\lambda\left(x_{k-1}-\varepsilon\right)},\right. \\
& G_{\lambda, k}=E_{W}^{\lambda x_{k-1}}\left\{\exp \left(-\xi_{k} \lambda^{-1 / \kappa} T_{\lambda\left(x_{k}-\varepsilon\right)}\right) ; T_{\lambda\left(x_{k}-\varepsilon\right)}>T_{\lambda\left(x_{k-1}-\varepsilon\right)},\right.
\end{aligned}
$$

then

$$
\begin{aligned}
& E_{W}^{0}\left\{\exp \left(-\sum_{k=1}^{n} \xi_{k} \lambda^{-1 / k}\left(T_{\lambda\left(x_{k}-\varepsilon\right)}-T_{\lambda x_{k-1}}\right)\right)\right\} \\
= & \prod_{k=1}^{n} E_{W}^{\lambda x_{k-1}}\left\{\exp \left(-\xi_{k} \lambda^{-1 / \kappa} T_{\lambda\left(x_{k}-\varepsilon\right)}\right)\right\} \\
= & \prod_{k=1}^{n}\left(F_{\lambda, k}+G_{\lambda, k}\right) .
\end{aligned}
$$

Making use of a trivial inequality $0 \leqq \prod_{k=1}^{n}\left(a_{k}+b_{k}\right)-\prod_{k=1}^{n} a_{k} \leqq \sum_{k=1}^{n} b_{k}$ which holds under the assumption that $a_{k}, b_{k} \geqq 0$ and $a_{k}+b_{k} \leqq 1(1 \leqq k \leqq n)$, we have

$$
\begin{aligned}
0 & \leqq E\left\{\prod_{k=1}^{n}\left(F_{\lambda, k}+G_{\lambda, k}\right)\right\}-E\left\{\prod_{k=1}^{n} F_{\lambda, k}\right\} \\
& \leqq \sum_{k=1}^{n} E\left\{G_{\lambda, k}\right\} \leqq \sum_{k=1}^{n} \mathscr{Q}^{\lambda x_{k-1}}\left\{T_{\lambda\left(x_{k}-\varepsilon\right)}>T_{\lambda\left(x_{k-1}-\varepsilon\right)}\right\} \\
& =\sum_{k=1}^{n} E\left\{\int_{\lambda x_{k-1}}^{\lambda\left(x_{k}-\varepsilon\right)} e^{W_{k}(x)} d x / \int_{\lambda\left(x_{k-1}-\varepsilon\right)}^{\lambda\left(x_{k}-\varepsilon\right)} e^{W_{k}(x)} d x\right\}
\end{aligned}
$$




$$
\begin{aligned}
& =\sum_{k=1}^{n} E\left\{\int_{0}^{\lambda\left(x_{k}-x_{k-1-\varepsilon)}\right.} e^{W_{k}\left(y+\lambda x_{k-1}\right)} d y / \int_{-\lambda \varepsilon}^{\lambda\left(x_{k}-x_{k}-1^{-\varepsilon}\right)} e^{W_{k}\left(y+\lambda x_{k-1}\right)} d y\right\} \\
& =\sum_{k=1}^{n} E\left\{\int_{0}^{\lambda\left(x_{k}-x_{k-1}-\varepsilon\right)} e^{W_{k}(y)} d y / \int_{-\lambda \varepsilon}^{\lambda\left(x_{k}-x_{k-1}-\varepsilon\right)} e^{W_{k}(y)} d y\right\} \\
& \quad \text { (since } W_{k} \text { has stationary increments) } \\
& \leqq n E\left\{\int_{0}^{\infty} e^{W_{k}(y)} d y / \int_{-\lambda \varepsilon}^{\infty} e^{W_{k}(y)} d y\right\} \rightarrow 0, \quad \lambda \rightarrow \infty .
\end{aligned}
$$

On the other hand it is easy to see that

$$
\begin{gathered}
E\left\{\prod_{k=1}^{n} F_{\lambda, k}\right\}=\prod_{k=1}^{n} E\left\{F_{\lambda, k}\right\}, \\
\lim _{\lambda \rightarrow \infty} E\left\{F_{\lambda, k}\right\}=\lim _{\lambda \rightarrow \infty} E\left\{F_{\lambda, k}+G_{\lambda, k}\right\} \\
=\lim _{\lambda \rightarrow \infty} \mathcal{E}^{0}\left\{\exp \left(-\xi_{k} \lambda^{-1 / k} T_{\lambda\left(x_{k}-x_{k-1-\varepsilon}\right)}\right)\right\} \\
=\exp \left\{-c\left(x_{k}-x_{k-1}-\varepsilon\right) \xi_{k}^{\kappa}\right\},
\end{gathered}
$$

the last equality being a consequence of (5). From (4.3) (4.6) we obtain (4.2). To derive (4.1) from (4.2) it is enough to notice that

$$
\begin{aligned}
0 \leqq & \mathcal{E}^{0}\left\{\exp \left(-\sum_{k=1}^{n} \xi_{k} \lambda^{-1 / \kappa}\left(T_{\lambda\left(x_{k}-\varepsilon\right)}-T_{\lambda x_{k-1}}\right)\right)\right\} \\
& -\mathcal{E}^{0}\left\{\exp \left(-\sum_{k=1}^{n} \xi_{k} \lambda^{-1 / \kappa}\left(T_{\lambda x_{k}}-T_{\lambda x_{k-1}}\right)\right)\right\} \\
\leqq & \mathcal{E}^{0}\left\{1-\exp \left(-\sum_{k=1}^{n} \xi_{k} \lambda^{-1 / \kappa}\left(T_{\lambda x_{k}}-T_{\lambda\left(x_{k}-\varepsilon\right)}\right)\right)\right\} \\
\leqq & \sum_{k=1}^{n} \mathcal{E}^{0}\left\{1-\exp \left(-\xi_{k} \lambda^{-1 / \kappa}\left(T_{\lambda x_{k}}-T_{\lambda\left(x_{k}-\varepsilon\right)}\right)\right)\right\} \\
= & \sum_{k=1}^{n} \mathcal{E}^{0}\left\{1-\exp \left(-\xi_{k} \lambda^{-1 / \kappa} T_{\lambda \varepsilon}\right)\right\} \\
\rightarrow & \sum_{k=1}^{n}\left\{1-\exp \left(-c \varepsilon \xi_{k}^{k}\right)\right\} \quad \text { as } \lambda \rightarrow \infty \\
\rightarrow & 0 \quad \text { as } \varepsilon \downarrow 0 .
\end{aligned}
$$

The proof of (ii) can be done in a way similar to (6). In fact, as in (3.33) we have for any $t_{k}>0, x_{k}>0(1 \leqq k \leqq n)$ and $y>0$

$$
\begin{aligned}
& \mathscr{P}^{0}\left\{T_{\lambda \boldsymbol{\kappa}_{x_{k}}} \geqq \lambda t_{k}, 1 \leqq k \leqq n\right\} \\
\leqq & \mathcal{P}^{0}\left\{\lambda^{-\kappa} \omega\left(\lambda t_{k}\right) \leqq x_{k}, 1 \leqq k \leqq n\right\} \\
\leqq & \mathscr{P}^{0}\left\{T_{\lambda \boldsymbol{\kappa} x_{k}+y} \geqq \lambda t_{k}, 1 \leqq k \leqq n\right\}+n \mathscr{P}^{0}\left\{\inf _{s \geq 0} \omega(s) \leqq-y\right\} .
\end{aligned}
$$


Letting $\lambda \uparrow \infty$ in the above we obtain

$$
\begin{aligned}
& \lim _{\lambda \rightarrow \infty} \mathscr{Q}^{0}\left\{\lambda^{-k} \omega\left(\lambda t_{k}\right) \leqq x_{k}, 1 \leqq k \leqq n\right\} \\
= & P\left\{L\left(x_{k}\right) \geqq t_{k}, 1 \leqq k \leqq n\right\}=P\left\{L^{-1}\left(t_{k}\right) \leqq x_{k}, 1 \leqq k \leqq n\right\} .
\end{aligned}
$$

\section{§. Proof of Theorem 1 in the case $\kappa=1$.}

Assume $\kappa=1$ and recall

$$
E_{W}^{0}\left\{e^{-\lambda T_{t}}\right\}=\exp \left\{-2 \lambda \int_{0}^{t} V_{\lambda}(s) d s\right\}, \text { a.s., }
$$

where $V_{\lambda}(t)=(2 \lambda)^{-1} U_{\lambda}(t)$ is a stationary diffusion process with generator

$$
\mathcal{L}_{\lambda}=\frac{d}{m_{\lambda}(d x)} \cdot d S_{\lambda}(\overline{x)},
$$

wherein

$$
S_{\lambda}(x)=\int_{0}^{x} \exp \left(\frac{2}{y}+4 \lambda y\right) d y, \quad m_{\lambda}(d x)=2 x^{-2} \exp \left(-\frac{2}{x}-4 \lambda x\right) d x .
$$

Once the following proposition is proved, (7) of Theorem 1 follows immediately from (5.1).

PROPOSITION 1. For any $\lambda>0$

$$
(x \log x)^{-1} \int_{0}^{x} V_{\lambda(x \log x)^{-1}(t) d t \rightarrow 2} \text { in probability as } x \rightarrow \infty .
$$

Before proving this proposition we prepare three lemmas. We put for $\xi>0$

$$
\begin{aligned}
& a_{\xi}=m_{\xi}\left(\boldsymbol{R}^{+}\right)^{-1} \int_{0}^{\infty} x m_{\xi}(d x), \\
& u_{\xi}(x)=\int_{0}^{x} d S_{\xi}(y) \int_{0}^{y}\left(z-a_{\xi}\right) m_{\xi}(d z) .
\end{aligned}
$$

LEMMA $6 . \quad a_{\xi} \sim-2 \log \xi$ as $\xi \downarrow 0$.

PROOF. $a_{\xi}$ can be expressed as $a_{\xi}=2 m_{\xi}\left(\boldsymbol{R}^{+}\right)^{-1}\left(I_{\xi}+I I_{\xi}\right)$ where

$$
I_{\xi}=\int_{0}^{N} x^{-1} \exp \left\{-2 x^{-1}-4 \xi x\right\} d x, \quad I I_{\xi}=\int_{N}^{\infty} x^{-1} \exp \left\{-2 x^{-1}-4 \xi x\right\} d x .
$$

For fixed $N>0 I_{\xi}$ remains bounded as $\xi \downarrow 0$ and $e^{-2 / N} I I_{\xi}^{\prime} \leqq I I_{\xi} \leqq I I_{\xi}^{\prime}$ where

$$
I I_{\xi}^{\prime}=\int_{N}^{\infty} x^{-1} e^{-4 \xi x} d x=\int_{4 \xi N}^{\infty} y^{-1} e^{-y} d y \sim-\log \xi
$$


as $\xi \downarrow 0$. Thus the lemma is proved since $m_{\xi}\left(\boldsymbol{R}^{+}\right)^{-1} \rightarrow 1$ as $\xi \downarrow 0$.

LEMMA 7. $\xi^{2} \int_{0}^{\infty} u_{\xi}(x)^{2} m_{\xi}(d x) \rightarrow 0$ as $\xi \downarrow 0$.

Proof. In what follows const. means a constant which is independent of $\xi$ but may vary from place to place. First we prove

$$
0<-u_{\xi}(x) \leqq \text { const. } x \log \frac{1}{\xi} \text { for } 0<x \leqq a_{\xi} .
$$

The restriction $0<x \leqq a_{\xi}$ implies

$$
\begin{aligned}
0<-u_{\xi}(x) & =2 \int_{0}^{x} \exp \left\{2 y^{-1}+4 \xi y\right\} d y \int_{0}^{y}\left(a_{\xi}-z\right) z^{-2} \exp \left\{-2 z^{-1}-4 \xi z\right\} d z \\
& \leqq 2 a_{\xi} \int_{0}^{x} \exp \left\{2 y^{-1}+4 \xi y\right\} d y \int_{0}^{y} z^{-2} \exp \left\{-2 z^{-1}-4 \xi z\right\} d z .
\end{aligned}
$$

Since $0<y<x<a_{\xi} \sim-2 \log \xi$ as $\xi \downarrow 0$, we have $0<\xi y<\xi a_{\xi} \rightarrow 0$ as $\xi \downarrow 0$. Therefore

$$
-u_{\xi}(x) \leqq \text { const. } \log \frac{1}{\xi} \int_{0}^{x} \exp \left\{2 y^{-1}\right\} d y \int_{0}^{y} z^{-2} \exp \left\{-2 z^{-1}\right\} d z \leqq \text { const. } x \log \frac{1}{\xi}
$$

as was to be proved. Next we prove

$$
0<-u_{\xi}(x) \leqq \text { const. } x \log \frac{1}{\xi}+\text { const. } \frac{1}{\xi} \log \frac{x}{a_{\xi}} \text { for } x>a_{\xi} .
$$

Since

$$
-\int_{0}^{y}\left(z-a_{\xi}\right) m_{\xi}(d z)=\int_{y}^{\infty}\left(z-a_{\xi}\right) m_{\xi}(d z),
$$

$-u_{\xi}(x)$ can be expressed as $-u_{\xi}(x)=2(I+I I)$, where

$$
\begin{aligned}
0<I & =2 \int_{0}^{a_{\xi}} \exp \left\{2 y^{-1}+4 \xi y\right\} d y \int_{0}^{y}\left(a_{\xi}-z\right) z^{-2} \exp \left\{-2 z^{-1}-4 \xi z\right\} d z \\
& \left.\leqq \text { const. } x \log \frac{1}{\xi} \quad \text { by } \underline{(5.3)}\right), \\
0<I I & =2 \int_{a_{\xi}}^{x} \exp \left\{2 y^{-1}+4 \xi y\right\} d y \int_{y}^{\infty}\left(z-a_{\xi}\right) z^{-2} \exp \left\{-2 z^{-1}-4 \xi z\right\} d z \\
& \leqq 2 \int_{a_{\xi}}^{x} \exp \left\{2 y^{-1}+4 \xi y\right\} d y \int_{y}^{\infty} z^{-1} \exp \{-4 \xi z\} d z .
\end{aligned}
$$

If we put $g(y)=\int_{y}^{\infty} z^{-1} e^{-4 \xi z} d z$, then 


$$
g(y)=\int_{4 \xi y}^{\infty} u^{-1} e^{-u} d u \leqq(4 \xi y)^{-1} e^{-4 \xi y},
$$

and hence

$$
\begin{aligned}
I I & \leqq 2 \int_{a_{\xi}}^{x} \exp \left\{2 y^{-1}+4 \xi y\right\} \cdot(4 \xi y)^{-1} e^{-4 \xi y} d y \\
& \leqq \text { const. } \frac{1}{\xi} \int_{a_{\xi}}^{x} \frac{d y}{y}=\text { const. } \frac{1}{\xi} \log \frac{x}{a_{\xi}} .
\end{aligned}
$$

This proves (5.4). We can now complete the proof of Lemma 7 as follows. From (5.3) and (5.4) we have

$$
\begin{aligned}
\xi^{2} \int_{0}^{\infty} u_{\xi}(x)^{2} m_{\xi}(d x) \leqq & \text { const. }\left(\xi \log \frac{1}{\xi}\right)^{2} \int_{0}^{a_{\xi}} \exp \left\{-2 x^{-1}-4 \xi x\right\} d x \\
& + \text { const. }\left(\xi \log \frac{1}{\xi}\right)^{2} \int_{a_{\xi}}^{\infty} \exp \left\{-2 x^{-1}-4 \xi x\right\} d x \\
& + \text { const. } \int_{a_{\xi}}^{\infty}\left(\log \frac{x}{a_{\xi}}\right)^{2} x^{-2} \exp \left\{-2 x^{-2}-4 \xi x\right\} d x \\
\leqq & \text { const. }\left(\xi \log \frac{1}{\xi}\right)^{2} \int_{0}^{\infty} e^{-4 \xi x} d x \\
& + \text { const. } \int_{a_{\xi}}^{\infty}\left(\log \frac{x}{a_{\xi}}\right)^{2} x^{-2} d x \\
= & \text { const. } \xi\left(\log \frac{1}{\xi}\right)^{2}+{ }^{-\frac{c o n s t}{a}} \int_{1}^{\infty}(\log y)^{2} y^{-2} d y \\
& \rightarrow 0 \quad \text { as } \xi \downarrow 0 .
\end{aligned}
$$

LEMMA 8. $\left(\log \frac{1}{\xi}\right)^{-1} \xi \int_{0}^{\infty}\left|u_{\xi}^{\prime}(x)\right|^{2} x^{2} m_{\xi}(d x) \rightarrow 0$ as $\xi \downarrow 0$.

Proof. First we prove

$$
0<-u_{\xi}^{\prime}(x) \leqq \text { const. } \log \frac{1}{\xi} \text { for } 0<x \leqq a_{\xi} .
$$

Under the restriction $0<x \leqq a_{\xi}$ we have

$$
\begin{aligned}
0 & <-u_{\xi}^{\prime}(x)=2 \exp \left\{2 x^{-1}+4 \xi x\right\} \int_{0}^{x}\left(a_{\xi}-y\right) y^{-2} \exp \left\{-2 y^{-1}-4 \xi y\right\} d y \\
& <2 a_{\xi} \exp \left\{2 x^{-1}+4 \xi x\right\} \int_{0}^{x} y^{-2} \exp \left\{-2 y^{-1}-4 \xi y\right\} d y \\
& \leqq \text { const. } \log \frac{1}{\xi} e^{2 / x} \int_{0}^{x} y^{-2} e^{-2 / y} d y=\text { const. } \log \frac{1}{\xi} .
\end{aligned}
$$

Next we prove 


$$
0<-u_{\xi}^{\prime}(x) \leqq \text { const. } e^{4 \xi x} \varphi(4 \xi x) \text { for } x>a_{\xi} \text {, }
$$

where

$$
\varphi(x)=\int_{x}^{\infty} y^{-1} e^{-y} d y
$$

In fact, if $x>a_{\xi}$ then

$$
\begin{aligned}
0<-u_{\xi}^{\prime}(x) & =2 \exp \left\{2 x^{-1}+4 \xi x\right\} \int_{x}^{\infty}\left(y-a_{\xi}\right) y^{-2} \exp \left\{-2 y^{-1}-4 \xi y\right\} d y \\
& \leqq \text { const. } e^{4 \xi x} \int_{x}^{\infty} y^{-1} e^{-4 \xi y} d y \\
& =\text { const. } e^{4 \xi x} \varphi(4 \xi x) .
\end{aligned}
$$

Now the proof of Lemma 8 is completed as follows.

$$
\begin{aligned}
& \left(\log \frac{1}{\xi}\right)^{-1} \xi \int_{0}^{a_{\xi}}\left|u_{\xi}^{\prime}(x)\right|^{2} x^{2} m_{\xi}(d x) \\
\leqq & \text { const. } \xi \log \frac{1}{\xi} \int_{0}^{a_{\xi}} \exp \left\{-2 x^{-1}-4 \xi x\right\} d x \quad \text { by (5.5)) } \\
\leqq & \text { const. } \xi \log \frac{1}{\xi} \cdot a_{\xi} \rightarrow 0 \quad \text { as } \xi \downarrow 0 .
\end{aligned}
$$

To estimate the integral over $\left(a_{\xi}, \infty\right)$ we notice that

$$
\varphi(x) \sim \begin{cases}e^{-x} / x & \text { as } x \rightarrow \infty, \\ \log \frac{1}{x} & \text { as } x \downarrow 0,\end{cases}
$$

and hence $e^{x} \varphi(x)^{2} \in L^{1}(0, \infty)$. Therefore

$$
\begin{aligned}
& \left(\log \frac{1}{\xi}\right)^{-1} \xi \int_{a_{\xi}}^{\infty}\left|u_{\xi}^{\prime}(x)\right|^{2} x^{2} m_{\xi}(d x) \\
\leqq & \text { const. } \left.\xi\left(\log \frac{1}{\xi}\right)^{-1} \int_{a_{\xi}}^{\infty} e^{4 \xi x} \varphi(4 \xi x)^{2} d x \quad \text { (by }(5.6)\right) \\
= & \text { const. }\left(\log \frac{1}{\xi}\right)^{-1} \int_{4 \xi a_{\xi}}^{\infty} e^{x} \varphi(x)^{2} d x \\
& \rightarrow 0 \quad \text { as } \xi \downarrow 0 .
\end{aligned}
$$

We now proceed to the proof of Proposition 1. Since $\mathcal{L}_{\xi} u_{\xi}=x-a_{\xi}$, an application of Itô's formula yields

$$
u_{\xi}\left(V_{\xi}(t)\right)-u_{\xi}\left(V_{\xi}(0)\right)=\int_{0}^{t} u_{\xi}^{\prime}\left(V_{\xi}(s)\right) V_{\xi}(s) d W(s)+\int_{0}^{t}\left(V_{\xi}(s)-a_{\xi}\right) d s .
$$


Putting $t=x, \xi=\lambda(x \log x)^{-1}$ and multiplying the both sides of the above by $(x \log x)^{-1}$ we have

$$
\begin{aligned}
& (x \log x)^{-1} \int_{0}^{x}\left(V_{\xi}(s)-a_{\xi}\right) d s \\
= & (x \log x)^{-1}\left\{u_{\xi}\left(V_{\xi}(x)\right)-u_{\xi}\left(V_{\xi}(0)\right)\right\}-(x \log x)^{-1} \int_{0}^{x} u_{\xi}^{\prime}\left(V_{\xi}(s)\right) V_{\xi}(s) d W(s) .
\end{aligned}
$$

The distribution of $V_{\xi}(s)$ is $c_{\xi} m_{\xi}(d x)$ where $c_{\xi}$ is the normalizing constant which tends to a finite value as $\xi \downarrow 0$. Therefore the second moment of the left hand side of $[5.7$ is dominated by

$$
\text { const. }(x \log x)^{-2} \int_{0}^{\infty} u_{\xi}(y)^{2} m_{\xi}(d y)+\text { const. }(x \log x)^{-2} x \int_{0}^{\infty}\left|u_{\xi}^{\prime}(y)\right|^{2} y^{2} m_{\xi}(d y),
$$

which tends to 0 as $x \rightarrow \infty$ by virtue of Lemma 7 and Lemma 8 because

$$
(x \log x)^{-2} x \sim \frac{1}{\lambda}\left(\log \frac{1}{\xi}\right)^{-1} \xi \text { as } x \rightarrow \infty .
$$

This combined with

$$
(x \log x)^{-1} \int_{0}^{x} a_{\xi} d s \rightarrow 2 \text { as } x \rightarrow \infty
$$

proves Proposition 1.

The assertion of (7) of Theorem 1 follows immediately from Proposition 1. The assertion (8) can be derived from (7) by a method similar to that used for deriving (6) in $\S 3$.

\section{§. Proof of Theorem 1 in the case $\kappa>1$.}

For any integer $k \geqq 1$ we put $\tau_{k}=T_{k}-T_{k-1}$. Then for any $\lambda_{1}, \lambda_{2}, \cdots, \lambda_{n} \geqq 0$ we have

$$
\begin{aligned}
E_{W}^{0}\left\{\exp \left(-\sum_{k=1}^{n} \lambda_{k} \tau_{k}\right)\right\} & =\prod_{k=1}^{n} E_{W}^{k-1}\left\{\exp \left(-\lambda_{k} \tau_{k}\right)\right\} \\
& =\prod_{k=1}^{n} f\left(\Gamma_{k-1} W, \lambda_{k}\right),
\end{aligned}
$$

where $f(W, \lambda)=E_{W}^{0}\left\{\exp \left(-\lambda T_{1}\right)\right\}$ and $\Gamma_{x}: \boldsymbol{W} \rightarrow \boldsymbol{W}$ (for fixed $x$ ) is defined by $\left(\Gamma_{x} W\right)(y)=W(x+y)-W(x)$ for any $y \in \boldsymbol{R}$. From (6.1) and the ergodicity of $\left\{\Gamma_{x}\right\}$ it follows that $\left\{\tau_{k}, k \geqq 1, \mathscr{P}^{0}\right\}$ is stationary and ergodic. Therefore

$$
\lim _{n \rightarrow \infty} \frac{T_{n}}{n}=\lim _{n \rightarrow \infty} \frac{\tau_{1}+\cdots+\tau_{n}}{n}=\mathcal{E}^{0}\left\{\tau_{1}\right\}, \quad \mathscr{P}^{0} \text {-a.s. }
$$


The condition $\kappa>1$ is equivalent to the finiteness of $\mathcal{E}^{0}\left\{\tau_{1}\right\}$ as will be seen below. First we compute $E_{W}^{0}\left\{T_{1}\right\}$; the result is

$$
E_{W}^{0}\left\{T_{1}\right\}=\int_{0}^{1} d S_{W}(x) \int_{-\infty}^{x} m_{W}(d y) .
$$

Therefore

$$
\begin{aligned}
\mathcal{E}^{0}\left\{\tau_{1}\right\} & =E\left\{W_{W}^{0}\left(T_{1}\right)\right\} \\
& =2 \int_{0}^{1} d x \int_{-\infty}^{x} E\left\{\exp \left\{W_{\kappa}(x)-W_{\kappa}(y)\right\}\right\} d y=\frac{4}{\kappa-1} .
\end{aligned}
$$

Thus (9) follows from (6.2). Next we prove (10), Clearly $\bar{\omega}(t) \rightarrow \infty$ as $t \uparrow \infty$ $\left(\mathscr{Q}^{0}\right.$-a.s. $)$ and for any $\varepsilon>0$

$$
\frac{T_{\bar{\omega}(t)}}{\bar{\omega}(t)} \leqq \frac{t}{\bar{\omega}(t)}<\frac{T_{\bar{\omega}(t)+\varepsilon}}{\bar{\omega}(t)}
$$

Letting $t \uparrow \infty$ in the above we obtain

$$
\lim _{t \rightarrow \infty} \frac{t}{\overline{\boldsymbol{\omega}}(t)}=\frac{4}{\kappa-1}, \quad \mathcal{Q}^{0} \text {-a.s. }
$$

which means that the left hand side of (10) equals $(\kappa-1) / 4$, a.s.. To prove that the second and the third terms of $(10)$ equal $(\kappa-1) / 4$, a.s., we put $\theta(n)=(1-\varepsilon)$ $\cdot(\kappa-1) n / 4$ for an integer $n \geqq 1$ and for $0<\varepsilon<1$. Then

$$
\begin{aligned}
& \mathcal{P}^{0}\left\{\inf _{s \geq T_{\theta(n)}} \omega(s)-\theta(n)<-\sqrt{n}\right\} \\
= & E\left\{P_{W}^{\theta(n)}\left(\inf _{s \geq 0} \omega(s)-\theta(n)<-\sqrt{n}\right)\right\} \\
= & \mathscr{P}^{0}\left\{\inf _{s \geqq 0} \omega(s)<-\sqrt{n}\right\} .
\end{aligned}
$$

The last term in the above is a general term of a convergent series by the result of [9]. Therefore an application of Borel-Cantelli's lemma yields

$$
\inf _{s \geq T_{\theta(n)}} \omega(s)-\theta(n) \geqq-\sqrt{n}
$$

for all sufficiently large $n, \mathscr{Q}^{0}$-a.s.. Since $T_{\theta(n)} / n \rightarrow 1-\varepsilon$ as $n \rightarrow \infty$ ( $\mathscr{Q}^{0}$-a.s.), (6.3) implies

$$
\inf _{s \geq n} \omega(s)-\theta(n) \geqq-\sqrt{n}
$$

for all sufficiently large $n, \mathscr{Q}^{0}$-a.s.. For $t>0$ we now take $n=n(t)$ such that $n \leqq t<n+1$. Then (6.4) implies 


$$
\theta(n)-\sqrt{n} \leqq \underline{\omega}(n) \leqq \underline{\omega}(t) \leqq \omega(t) \leqq \bar{\omega}(t)
$$

for all sufficiently large $t, \mathscr{P}^{0}$-a.s.. Dividing (6.5) by $t$ and then letting $t \uparrow \infty$ we finally see that the second and the third terms of $(10)$ equal $(\kappa-1) / 4, \mathscr{P}^{0}$-a.s.

\section{$\S 7$. Remark to the case $\kappa=0$.}

A limit theorem concerning $\bar{\omega}(t)=\max \{\omega(s): 0 \leqq s \leqq t\}$ was obtained by Kotani (1988), Ogura (1989) and Kawazu-Tanaka (1989) independently and by different methods (proofs were unpublished). The result is as follows. Let $t>0$ and put

$$
\begin{array}{ll}
W^{\#}(x)=W(x)-\min _{[x \wedge 0, x \vee 0]} W, & x \in \boldsymbol{R}, \\
d_{t}^{+}=\min \left\{x>0: W^{\#}(x)=t\right\}, & V_{t}^{+}=\min _{\left[0, d_{t}^{+}\right]} W, \\
d_{t}^{-}=\max \left\{x<0: W^{\#}(x)=t\right\}, & V_{t}^{-}=\min _{\left[d_{t}^{-}, 0\right]} W,
\end{array}
$$

and define $b_{t}^{+}$and $b_{t}^{-}$by $W\left(b_{t}^{+}\right)=V_{t}^{ \pm}$(such $b_{t}^{ \pm}$are uniquely determined $P$-a.s. for each fixed $t>0$ ). Let

$$
\begin{aligned}
& M_{t}^{+}=\max _{\left[0, b_{t}^{+}\right]} W, \quad J_{t}^{+}=M_{t}^{+} \vee\left(V_{t}^{+}+t\right), \\
& M_{\bar{t}}^{-}=\max _{\left[b_{t}^{-}, 0\right]} W, \quad J_{t}^{-}=M_{t}^{-} \vee\left(V_{t}^{-}+t\right),
\end{aligned}
$$

and finally define $b_{t}^{\#}$ by

$$
b_{t}^{\#}= \begin{cases}\min \left\{x>0: W^{\#}(x)=t\right\} & \text { if } J_{t}^{+}<J_{t}^{-}, \\ \min \left\{x>0: W(x)=J_{t}^{-}\right\} & \text {if } J_{t}^{+}>J_{t}^{-} .\end{cases}
$$

Then the process $\left\{\lambda^{-2} \bar{\omega}\left(e^{\lambda t}\right), t>0, \mathscr{P}^{0}\right\}$ converges to $\left\{b_{t}^{\#}, t>0, P\right\}$ as $\lambda \rightarrow \infty$ in the sense of convergence of finite dimensional distributions. In particular, $(\log t)^{-2} \bar{\omega}(t)$ converges in law to $b_{1}^{\#}$ as $t \rightarrow \infty$ and

$$
E\left\{e^{-\xi b_{1}^{\#}}\right\}=\int_{0}^{1} E_{+}^{x}\left\{e^{-\xi T_{1}}\right\} d x, \quad \xi \geqq 0,
$$

where $E_{+}^{x}$ and $T_{1}$ denote the expectation and the first hitting time to 1 , respectively, for the reflecting Brownian motion on $[0, \infty)$ starting at $x$.

The corresponding result in the case of random walks on $\{0,1,2, \cdots\}$ with reflecting barrier at 0 was obtained by Golosov [2].

\section{References}

[1] T. Brox, A one-dimensional diffusion process in a Wiener medium, Ann. Probab., 14 (1986), 1206-1218. 
[2] A. O. Golosov, Limit distributions for random walks in random environments, Soviet Math. Dokl., 28 (1983), 18-22.

[3] A. O. Golosov, Localization of random walks in one-dimensional random environments, Comm. Math. Phys., 92 (1984), 491-506.

[4] K. Itô, Stochastic Processes II, Iwanami, 1958, (in Japanese).

[5] K. Itô and M. Nisio, On stationary solutions of a stochastic differential equation, J. Math. Kyoto Univ., 4-1 (1964), 1-75.

[6] I.S. Kac and M.G. Krein, On the spectral functions of the string, Amer. Math. Soc. Transl. (2), 103 (1974), 19-102.

[7] Y. Kasahara, Spectral theory of generalized second order differential operators and its applications to Markov processes, Japan J. Math., 1 (1975), 67-84.

[8] K. Kawazu, Y. Tamura and H. Tanaka, Localization of diffusion processes in onedimensional random environment, J. Math. Soc. Japan, 44 (1992), 515-550.

[9] K. Kawazu and H. Tanaka, On the maximum of a diffusion process in a drifted Brownian environment, In Séminaire de Probabilités, (eds. J. Azéma et al.), Lecture Notes in Math., 1557, Springer-Verlag, 1993, 78-85.

[10] H. Kesten, M. V. Kozlov and F. Spitzer, A limit law for random walk in a random environment, Compositio Math., 30 (1975), 145-168.

[11] H. Kesten, The limit distribution of Sinai's random walk in random environment, Physica, 138A (1986), 299-309.

[12] F.B. Knight, Characterization of the Lévy measure of inverse local times of gap diffusions, In Seminar on Stochastic Processes, Birkhäuser, 1981, 53-78.

[13] S. Kotani and S. Watanabe, Krein's spectral theory of strings and generalized diffusion processes, In Functional Analysis in Markov Processes, (ed. M. Fukushima), Lecture Notes in Math., 923, Springer-Verlag, 1982, 235-259.

[14] M. V. Kozlov, A random walk on the line with stochastic structure, Theory Probab. Appl., 18 (1973), 406-408.

[15] Y. Ogura, One-dimensional bi-generalized diffusion processes, J. Math. Soc. Japan, 41 (1989), 213-242.

[16] S. Schumacher, Diffusions with random coefficients, Particle Systems, Random Media and Large Deviations, Contemp. Math., 41 (1985), 351-356.

[17] Ya.G. Sinai, The limiting behavior of a one-dimensional random walk in a random medium, Theory Probab. Appl., 27 (1982), 256-268.

[18] F. Solomon, Random walks in a random environment, Ann. Probab., 3 (1975), 1-31.

[19] H. Tanaka, Localization of a diffusion process in a one-dimensional Brownian environment, Comm. Pure Appl. Math., XLVII (1994), 755-766.

[20] S. Watanabe, On time inversion of one-dimensional diffusion processes, Z. Wahrscheinlichtkeitstheorie verw. Gebiete, 31 (1975), 115-124.

\author{
Kiyoshi KawazU \\ Department of Mathematics \\ Faculty of Education \\ Yamaguchi University \\ Yoshida, Yamaguchi 753 \\ Japan
}

\author{
Hiroshi TANAKa \\ Department of Mathematics \\ Faculty of Science and Technology \\ Keio University \\ Hiyoshi, Yokohama 223 \\ Japan
}

